\title{
Rotational diffusion may govern the rheology of magnetic suspensions
}

\author{
P. Kuzhir ${ }^{1}$, C. Magnet ${ }^{1}$, G. Bossis ${ }^{1}$, A. Meunier ${ }^{1}$, V. Bashtovoi ${ }^{2}$ \\ ${ }^{1}$ Laboratory of Condensed Matter Physics, University of Nice $\square$ Sophia Antipolis, Parc Valrose 06108 Nice \\ Cedex 2 France \\ ${ }^{2}$ Belarussian National Technical University, 65, Prospekt Nezavisimosti, 220013 Minsk, Belarus
}

\section{Synopsis}

This paper is focused on the theoretical modeling of the rheological properties of the magnetic suspensions in shear flows under an external magnetic field aligned with the streamlines. The conventional theory postulates that the field-induced aggregates of magnetic particles are highly anisotropic and aligned with the flow direction. Therefore, no substantial variation in suspension viscosity would be expected in the presence of field. However, experiments reveal a strong Bingham rheological behavior of the suspensions with a dynamic yield stress of the same order of magnitude that the one measured in the magnetic fields perpendicular to the flow. We explain the high level of shear stress, generated in longitudinal magnetic fields, by stochastic rotary oscillations of the aggregates caused by many-body magnetic interactions with neighboring aggregates. The inter-aggregate interactions are accounted for by an effective rotational diffusion process with a diffusion constant proportional to the mean square interaction torque $\square$ a net magnetic torque exerted to a given aggregate by all the neighboring aggregates. Using the equations for the orientation tensors coupled with the balance of forces acting on aggregates, we find the orientation distribution and size of the aggregates as function of the magnetic field intensity and shear rate. Our theory, developed for semi-dilute regime, reproduces the Bingham behavior observed experimentally and fits the 
experimental data reasonably well in a wide range of particle concentrations. We find that the yield stress increases quadratically with the magnetic field strength and exhibits a cubic growth with respect to the particle volume fraction. A part from resolving a particular rheological problem, the new concept of magnetically induced diffusion reveals the importance of long-range non-hydrodynamic interactions in the rotary diffusion process and could probably contribute to the understanding of this process in other concentrated systems subject to non-hydrodynamic interactions.

\section{Introduction}

Non-Brownian magnetic dispersions, conventionally called magnetorheological (MR) fluids, are suspensions of magnetized micron-sized particles in a dispersing liquid. When an external magnetic field is applied, the particles acquire magnetic moments, attract to each other due to dipolar forces and form anisotropic aggregates aligned preferably with the magnetic field direction. Thus, upon a field application MR fluids undergo a reversible jamming responsible for a several order of magnitude increase in effective viscosity and appearance of a yield stress $\square$ threshold mechanical stress required for onset of flow [Ginder (1998)]. This phenomenon, referred to as magnetorheological effect, is being effectively used in numerous smart engineering applications [Carlson et al. (1996), Kordonski and Jacobs (1996)]. The coupling between field-induced structuring and hydrodynamic interactions gives rise to various striking non-linear phenomena, such as flow-induced shear banding and structural transitions [Cutillas et al. (1998), Volkova et al. (1999)], formation of honeycomb and foam structures [Martin et al. (2004)]. Extensive reviews on microstructure and rheology of MR fluids are given by Shulman and Kordonski (1982), Ginder (1998), Bossis et al. (2002a).

Most of the studies in mechanics of magnetic dispersions were focused on their flows in the presence of the magnetic field perpendicular to the flow geometry $\square$ presumably, the case of 
the largest practical interest. At such conditions, macroscopic mechanical behavior of the suspension followed, most often, a Bingham behavior with the shear stress being a sum of a solvent contribution $\eta_{0} \dot{\gamma}$ and a field-dependent aggregate stress, $\sigma_{Y}$, constant with shear rate, $\dot{\gamma}$, namely $\sigma=\sigma_{Y}+\eta_{0} \dot{\gamma}$, with $\eta_{0}$ being the solvent viscosity. A number of theoretical models has been proposed to describe such behavior [Shulman et al. (1986), Martin and Anderson (1996), Zubarev and Iskakova (2006)] but all of them give the similar explanation for the independence of the aggregate stress on shear rate. First, this stress scales roughly as $\eta_{0} \dot{\gamma} r_{e}^{2}$, with $r_{e}$ being the ratio of major to minor aggregate axes, called aspect ratio. Second, the aggregate length decreases with the increasing shear rate because of destructive action of hydrodynamic forces $\left(r_{e}^{2} \propto \dot{\gamma}^{-1}\right)$, therefore, shear rate vanishes in the aggregate stress and the latter is referred to as dynamic yield stress.

In magnetic fields parallel to channel walls, the particle aggregates are expected to be oriented along the stream-lines and be (in theory) infinitely long because they are not subject to tensile hydrodynamic forces. At such conditions, the suspension should undergo a Newtonian behavior with a viscosity of the order of magnitude of the solvent viscosity. However the stress level, developed in parallel fields, is relatively high and the suspension develops a strong Bingham behavior, which does not corroborate with the assumption of alignment of aggregates in flow direction. In particular, Shulman and Kordonsky (1982) and Kuzhir et al. (2003) have found that, in the pipe flows with a longitudinal magnetic field, concentrated magnetic suspensions developed a dynamic yield stress, which was only a few times smaller than the one in a perpendicular field. Takimoto et al. (1999) have reported a similar effect in a simple shear flow between two parallel plates. Bossis et al. (2002-b) have studied a shear flow of an MR fluid between two concentric cylinders in the presence of an axial magnetic field parallel to the fluid vorticity. In this geometry, the aggregates should be, a priori, stably 
aligned in the vorticity direction and should not cause significant viscous dissipation. Nevertheless, the MR fluid showed again a Bingham behavior with a yield stress almost the same as the one in the case of a magnetic field applied perpendicularly to the walls. Until now, none of these effects have been clearly explained or modeled. Furthermore, the concentration dependence of the observed MR effect has never been reported.

The scope of the present paper is to provide new experimental results and also a theoretical explanation of the appearance of large Bingham stress in longitudinal magnetic fields and develop a theoretical model predicting both the field- and concentration dependencies of this stress. Obviously, high mechanical stresses may only appear if the MR structures are misaligned relative to the fluid streamlines. For instance, if aggregate rotation is restricted to the shear plane, the aggregate shear stress scales as $\sigma \propto \eta_{0} \dot{\gamma} r_{e}^{2}<\cos ^{2} \theta \sin ^{2} \theta>$ [Batchelor (1971)], with $\theta$ being an angle between the aggregates and the streamlines, angle brackets denote averaging over all possible orientations. Thus, even a small angle deviation of aggregate orientation from the flow direction may generate a non-negligible stress, if the aggregate aspect ratio is high. Contrary to flow-aligned aggregates, a misaligned aggregate should have a large but finite length defined by the equilibrium between the tensile hydrodynamic force and the magnetic cohesive force. Thus, the aggregate aspect ratio is expected to follow the same shear rate dependence as in the case of the perpendicular magnetic field, $r_{e}^{2} \propto \dot{\gamma}^{-1}$. This condition, verified by our theory [cf. Section II], could explain the appearance of the dynamic yield stress in longitudinal fields. The main question now is what mechanism can be responsible for aggregate misalignment. The main hypothesis of the present study is that the aggregates can deviate from their orientation along the streamlines because of magnetic dipole interactions with the neighboring aggregates. Since the aggregates are randomly spaced in the suspension, under shear flow, they will change their mutual 
positions and orientations in irregular way. Together with many-body interactions, this may cause a stochastic variation in dipolar forces and torques experienced by the aggregates and could produce some fluctuations in their orientations. This process can be regarded as a magnetically induced rotational diffusion of aggregates, by analogy with Brownian rotational diffusion [Van de Ven (1989)] or flow-induced rotational diffusion of elongated particles caused by their collisions or short-range hydrodynamic interactions in sheared suspensions [Folgar and Tucker (1984), Férec et al. (2009)] or granular media [Otto et al. (2006)]. Stochastic torques coming from many-body magnetic interactions tend to randomize the aggregate orientation, while a shear flow and a restoring magnetic torque, exerted to aggregates by an external field, tend to align the aggregates with the flow. So, the fluctuations in aggregate orientation are not necessarily large and might not lead to collisions. In this case, we shall deal with a weak rotational diffusion caused solely by long-range dipole interactions. In support of this hypothesis, weak orientation fluctuations have recently been observed in experiments on kinetics of aggregation of diluted magnetic suspensions $\square$

Incorporating this new rotational diffusion mechanism into standard micromechanical models of MR fluids, we hope to give, for the first time, correct quantitative estimations of the Bingham stresses developed in longitudinal magnetic fields. In particular, in this paper, we consider theoretically a simple shear flow of a magnetic suspension under an external magnetic field collinear with the fluid velocity. Dynamics of aggregates and suspension viscoelastic properties are determined by means of the slender body theory [Batchelor (1970, 1971)] with taking into account magnetically induced rotational diffusion and long-range hydrodynamic interactions [Shaqfeh and Fredrickson (1990)]. In order to validate our theory, we perform a detailed experimental study of this flow using a capillary rheometer and

\footnotetext{
${ }^{\dagger}$ These experiments are currently being carried out in our research group and have not been published yet.
} 
compare experimental stress-vs.-field and stress-vs.-concentration dependencies with the predicted ones. Even though, we apply our theory to a particular problem of the magnetorheology, we hope it may be used for a more general purpose of understanding the role of long-range non-hydrodynamic interactions in rotational diffusion observed in particulate systems, when interparticle collisions are not mandatory.

This paper is organized as follows. In the next section II, we describe in details our theoretical model and give the final expressions for suspension viscoelastic properties. Experimental technique and procedures is described in the Section III. Both theoretical and experimental results are reported and compared in the Section IV. Finally, in the Section V, we outline the main conclusions of this work and some ideas for further improvement of the present model.

\section{Theory}

Let us consider a simple shear flow of a semi-dilute MR fluid with a linear velocity profile, $\mathbf{v}=\left(\dot{\gamma} x_{2}, 0,0\right)$, as depicted in Fig. 1. An external magnetic field, of intensity $\mathbf{H}$, is oriented along the fluid streamlines, i.e. along the $\mathrm{O} x_{1}$-axis. We are looking for the shear stress, $\sigma_{12}$, developed in the suspension as function of the shear rate, $\dot{\gamma}$, and magnetic field strength $H$. 


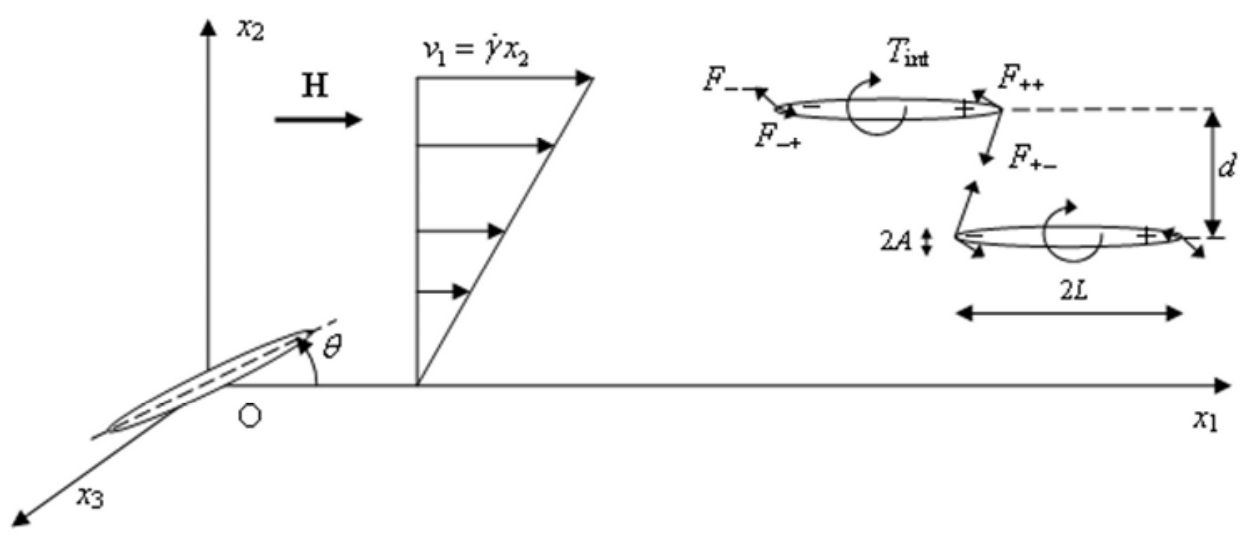

Fig.1. Geometry of the simple shear flow in the presence of the longitudinal magnetic field. A sketch of the forces acting between two aggregates is presented on the right part of the figure: $F_{+-}, F_{++}, F_{--}$and $F_{-+}$stand for the forces between each pair of poles of two neighboring aggregates. These forces induce a so-called interaction torque $T_{\text {int }}$ on both aggregates that makes them turn in the clockwise direction. In shear flow, the upper aggregate moves faster than the lower aggregate, the forces between poles vary as the aggregates displace. The interaction torque also varies and changes its direction when the upper aggregate overruns the lower one; this make them turn in the opposite direction. Thus, when the upper aggregate displaces approximately by two times its length, both aggregates will perform one angular oscillation. In the presence of many aggregates moving at different velocities the dipolar forces and interaction torques will vary stochastically and will result in stochastic angular oscillations of aggregates.

The following assumptions are introduced:

1. In the presence of the magnetic field, the particles are supposed to form long cylindrical aggregates aligned with the field direction, as usually observed in quiescent suspensions [Bossis et al. (2002-a)]. As already stated, the aggregates can deviate from their initial orientation because of magnetic interactions with the neighboring aggregates. Under shear flow, random mutual positions of aggregates with respect to each other will cause a stochastic variation in dipolar forces and torques and thus will produce some fluctuations in their orientations. Since the aggregates are subject to multi-body magnetic interactions, their rotational dynamics is a complex phenomenon required a detailed study by numerical simulations. At the present stage, we propose to describe this dynamics by a rotational diffusion process, which is supposed to be Gaussian delta-correlated process, similar to the Brownian rotational motion. The fluctuations of aggregate orientation can be seen as a random walk when the aggregates perform irregular jumps with the mean amplitude $\Delta \theta$ and 
the mean jump duration $\Delta t$. According to the random walk model [Van de Ven (1989)], the rotational diffusion constant scales as

$$
D_{r} \sim \frac{(\Delta \theta)^{2}}{\Delta t} \approx\left\langle\omega^{2}\right\rangle \Delta t
$$

where $\left\langle\omega^{2}\right\rangle$ is the mean square angular velocity of the aggregates. Note that, in the case of classical Brownian motion, the formula (1) gives the correct result for the rotational diffusion constant of Brownian particles up to a numerical constant. Doi and Edwards (1986) have also used the same reasoning [Eq. (1)] in their tube model for rotational diffusion of semi-dilute polymer solutions.

2. In our case, the mean jump duration can be estimated by considering the mutual displacement of two neighboring aggregates in shear flow. Suppose two aggregates displace parallel to each other along the streamlines spaced at the distance $d$ (Fig.1). The upper aggregate moves faster than the lower one, so, both aggregates will perform one angular oscillation when the upper aggregate overtakes the lower one and has moved by the distance $4 L$ relatively to its neighbor. The mean jump duration corresponds approximately to a halfperiod of the aggregate oscillation: $\Delta t \sim 2 L /(\dot{\gamma} d)$.

3. The amplitude of orientational fluctuations is measured by a torque, $T_{\text {int }}$, created by manybody magnetic interactions with neighboring aggregates and called hereinafter interaction torque. The mean square angular velocity of the stochastic motion of aggregates can be estimated as $\left\langle\omega^{2}\right\rangle \approx\left\langle T_{\mathrm{int}}{ }^{2}\right\rangle / f_{r}{ }^{2}$, with $\left\langle T_{\mathrm{int}}{ }^{2}\right\rangle$ being the mean square interaction torque and $f_{r}$ a rotational friction coefficient. Thus, the expression (1) for rotational diffusion constant reads

$$
D_{r} \sim \frac{\left\langle T_{\mathrm{int}}{ }^{2}\right\rangle}{f_{r}^{2}} \cdot \frac{2 L}{\dot{\gamma} d}
$$


4. The aggregates are considered to be dipoles with both poles situated on their extremities [Fig. 1]. Thus, the interaction force between two aggregates is the sum of four forces acting between the pair of poles of the two aggregates. For the estimation of the mean square interaction torque, $\left\langle T_{\mathrm{int}}{ }^{2}\right\rangle$ created by these forces, we can consider a particular case of the aggregate mutual position shown on the right part of Fig. 1. We consider that the upper aggregate displaces above the lower aggregate with the relative velocity $\dot{\gamma} d$, and we neglect the angular deviation of the aggregates $\square$ orientation from the flow direction. The interaction torque acting on the upper aggregate is induced by four forces of interaction between the poles of both aggregates, $\quad F_{+-}, \quad F_{++}, \quad F_{--}, \quad F_{-+} \quad$ [Fig.1], namely, $T_{\text {int }}=\left(F_{+-}{ }^{\perp}-F_{++}{ }^{\perp}+F_{--}{ }^{\perp}-F_{-+}{ }^{\perp}\right) L$, where the superscript $\square \perp \square$ denotes the force component perpendicular to the aggregate axis. The expression for the forces of interactions between poles reads [Rosensweig (1985)]: $F_{i}=p^{2} /\left(4 \pi \mu_{0} r_{i}^{2}\right)$, consequently, $F_{i}^{\perp}=p^{2} d /\left(4 \pi \mu_{0} r_{i}^{3}\right)$, where $i=+-,++,--,-+; r_{i}$ is the pole-to-pole distance, $p=m /(2 L)$ is the intensity of the magnetic pole, $\mu_{0}=4 \pi \cdot 10^{-7} \mathrm{H} / \mathrm{m}$ is the magnetic constant, $m=\mu_{0} M_{a} V_{a}$ is the aggregate magnetic moment induced by an external magnetic field of intensity $H, M_{a}=\chi_{a} H$ is the aggregate magnetization, $\chi_{a}$ is the aggregate magnetic susceptibility and $V_{a}=2 \pi A^{2} L$ is the aggregate volume with $A$ being the aggregate radius. Squaring the interaction torque and averaging the result over the position of the upper aggregate along the flow direction (over $x_{1}$ coordinate for the aggregate displacement of the order of its length) we obtain that the mean square torque scales as

$$
\left\langle T_{\mathrm{int}}{ }^{2}\right\rangle \sim T_{\mathrm{int} 0}{ }^{2} \cdot \frac{d}{L}
$$


where $T_{\text {int } 0}=p^{2} L /\left(4 \pi \mu_{0} d^{2}\right) \sim \mu_{0} \chi_{a}^{2} H^{2} A^{4} L / d^{2}$ is the maximal absolute value of the interaction torque for the mutual position of the aggregates, at which the front of the upper aggregate is at the same position $x_{1}$ as the back of the lower aggregate. The mean distance $d$ between thin (aspect ratio $r_{e}=L / A \gg 1$ ) quasi-aligned aggregates can be estimated as $d \sim A\left(\Phi / \Phi_{a}\right)^{-1 / 2}$, where the ratio $\Phi / \Phi_{a}$ stands for the volume fraction of porous aggregates in the suspension, with $\Phi$ being the volume fraction of particles in the suspension and $\Phi_{a}$ the volume fraction of particles inside the aggregates. Performing the necessary substitutions, we get the following expression for the mean square interaction torque, up to a numerical constant:

$$
\left\langle T_{\mathrm{int}}{ }^{2}\right\rangle \sim\left(\frac{\Phi}{\Phi_{a}} \mu_{0} \chi_{a}^{2} H^{2} V_{a}\right)^{2} \cdot \frac{d}{L}
$$

5. We neglect eventual collisions between aggregates and short-range hydrodynamic interactions but account for their long-range hydrodynamic interaction according to the theory of Shaqfeh and Fredrickson (1990), which leads to the following expressions for the linear density of hydrodynamic force on slender aggregates and for their rotational friction coefficient:

$$
\begin{gathered}
f_{\|}=\frac{2 \pi \eta_{0}}{\ln (\xi)}\left(v^{\infty}{ }_{\|}-v_{\|}\right), f_{\perp}=\frac{4 \pi \eta_{0}}{\ln (\xi)}\left(v^{\infty}{ }_{\perp}-v_{\perp}\right) \\
f_{r}=\frac{8 \pi \eta_{0} L^{3}}{3 \ln (\xi)},
\end{gathered}
$$

where $\eta_{0}$ is the viscosity of a Newtonian liquid-carrier of the MR fluid, $\xi$ is the screening length normalized by the aggregate radius $A$ and is a logarithmic function of $\Phi / \Phi_{a}, \mathbf{v}^{\infty}$ and $\mathbf{v}$ are, respectively, velocities of the undisturbed flow and of the aggregate at a given position 
along its major axis, subscripts $\square \square$ and $\square \square \square$ stand for the force and velocity components along the major and the minor aggregate axes, respectively. Strictly speaking this approach can be applied safely for the semi-dilute concentration limit $\Phi / \Phi_{a} \ll r_{e}^{-1}$ [Shaqfeh and Fredrickson (1990)], which is not satisfied for our experimental systems. However, in suspensions of quasi-aligned aggregates with presumably small collision rate, the short range hydrodynamic forces are expected to give a non-negligible but not decisive contribution to overall forces and torques experienced by aggregates. Therefore, the semi-dilute limit approach is expected to give correct estimations for the hydrodynamic forces and stresses. Substituting Eqs. (4) and (6) into Eq. (2), we obtain the final expression for the rotational diffusion constant, up to some numerical constant $\alpha$ :

$$
D_{r}=\alpha\left(\frac{\Phi}{\Phi_{a}} \frac{\mu_{0} \chi_{a}{ }^{2} H^{2}}{\eta_{0} \beta}\right)^{2} \frac{1}{\dot{\gamma}}
$$

where $\beta=4 r_{e}^{2} /[3 \ln (\xi)]$ is a form factor.

6. Note that both the mean square interaction torque $\left\langle T_{\text {int }}{ }^{2}\right\rangle$ [Eq. (4)] and the mean jump duration $\Delta t$ were estimated in the limit of pair magnetic interactions between two aggregates and without taking into account angular deviations of aggregates from the flow direction. These approximations could affect the value of the diffusion constant. Therefore, the numerical factor $\alpha$ in the expression (7) can be seen as a correction factor for many-body interactions and is called hereinafter interaction constant. The constant $\alpha$ is taken as a free parameter of our model.

7. In order to evaluate the suspension stress, we must first determine the orientation distribution of aggregates, or rather the second and the fourth moments of the distribution 
function, $\left\langle e_{i} e_{k}\right\rangle$ and $\left\langle e_{i} e_{k} e_{l} e_{m}\right\rangle$, where $\mathbf{e}$ is the unit vector along the aggregate major axis and $e_{i}$ is its component along the axis $\mathrm{O} x_{i}, i=1,2,3$. The common way of solving this problem consists of two steps: (i) determination of the angular distribution function by integration of the convective-diffusion equation [Van de Ven (1989)] and (ii) averaging the moments of the distribution function over all possible orientations. The alternative way allows direct determination of the desired quantities $\left\langle e_{i} e_{k}\right\rangle$ and $\left\langle e_{i} e_{k} e_{l} e_{m}\right\rangle$ by solving a set of simple equations describing temporal evolution of the statistical moments $\left\langle e_{i} e_{k}\right\rangle$. These equations are simply obtained from the convective-diffusion equation by multiplying it by $3\left\langle e_{i} e_{k}\right\rangle-\delta_{i k}$ (with $\delta_{i k}$ being the Kronecker delta) and averaging over all possible orientations [Doi and Edwards (1986), Pokrovskiy (1978)]. The first method gives more rigorous results but requires numerical solution of the convective-diffusion equation. The second method gives simple approximate solutions but requires some closure approximation concerning a relationship between the fourth and the second moments of the distribution function: $\left\langle e_{i} e_{k} e_{l} e_{m}\right\rangle=f\left(\left\langle e_{i} e_{k}\right\rangle\right)$. A rigorous numerical simulation would be useless, given the level of approximation used for the determination of the diffusion coefficient, therefore, we choose the second method of determination of the statistical moments using the evolution equations for $\left\langle e_{i} e_{k}\right\rangle$, given, in the most general form, by Pokrovskiy (1978) for axisymmetric particles subject to both shear flow and external torques. In the case of slender aggregates with hydrodynamic screening, this expression takes the following form:

$$
\begin{aligned}
& \frac{d\left\langle e_{i} e_{k}\right\rangle}{d t}=\left[\omega_{i l}\left\langle e_{l} e_{k}\right\rangle-\left\langle e_{i} e_{l}\right\rangle \omega_{l k}\right]+\left[\gamma_{i l}\left\langle e_{l} e_{k}\right\rangle+\left\langle e_{i} e_{l}\right\rangle \gamma_{l k}\right]-2\left\langle e_{i} e_{k} e_{l} e_{m}\right\rangle \gamma_{l m} \\
& -\frac{\mu_{0} H^{2}}{\eta_{0} \beta} \frac{\chi_{a}^{2}}{2+\chi_{a}}\left[\left\langle e_{i} e_{l} T^{m}{ }_{l k}\right\rangle+\left\langle e_{k} e_{l} T^{m}{ }_{l i}\right\rangle\right]+2 D_{r}\left[\delta_{i k}-3\left\langle e_{i} e_{k}\right\rangle\right]
\end{aligned}
$$


where $t$ is time, $\gamma_{i k}=(1 / 2) \cdot\left(\partial v_{i} / \partial x_{k}+\partial v_{k} / \partial x_{i}\right)$ and $\omega_{i k}=(1 / 2) \cdot\left(\partial v_{i} / \partial x_{k}-\partial v_{k} / \partial x_{i}\right)$ are rateof-strain and vorticity tensors, respectively, $\mathbf{T}^{m}$ is the magnetic torque applied to aggregates by the external magnetic field. For slender porous magnetic aggregates this torque is given by the following expression:

$$
T^{m}{ }_{i k}=\mu_{0} H^{2} \frac{\chi_{a}{ }^{2}}{2+\chi_{a}} V_{a}\left[h_{i} h_{l} e_{l} e_{k}-h_{k} h_{l} e_{l} e_{i}\right],
$$

with $\mathbf{h}=\mathbf{H} / H$ being a unit vector oriented along the magnetic field direction.

8. To solve the equation (8) with respect to $\left\langle e_{i} e_{k}\right\rangle$, we introduce the quadratic closure approximation, first postulated by Doi and Edwards (1986) when studying the dynamics of rigid rod-like polymers:

$$
\left\langle e_{i} e_{k} e_{l} e_{m}\right\rangle \equiv\left\langle e_{i} e_{k}\right\rangle\left\langle e_{l} e_{m}\right\rangle
$$

This expression becomes exact in the limit of perfect alignment of rod-like particles (or aggregates). In the case of non-perfect alignment, the exactness of this approximation decreases with decrease in degree of alignment of particles. So, the Eq. (10) can be applied, with a presumably small uncertainty, for our case of magnetic aggregates strongly aligned by shear and external magnetic fields. A variety of other closure approximations has been proposed in literature for non-Brownian fiber suspensions, liquid crystals, rigid polymers [Hinch and Leal (1976), Advani and Tucker (1987), Cintra and Tucker (1995), Chaubal and Leal (1998), Grosso et al. (2000), Chung and Kwon (2001)]. In our particular case, the choice of the closure approximation will not change qualitatively the final result $\square$ appearance of the dynamic yield stress in the longitudinal magnetic fields; it may only affect its numerical value. Testing different closure approximations is not of the scope of our paper; we restrict our analysis to the simplest approximation $\square$ the quadratic closure (10) $\square$ and focus our 
attention on the mechanism of the appearance of the yield stress rather than on the rigorous prediction of its numerical value.

9. The system of equations (7) $\square(10)$ is not yet closed because it contains another unknown quantity $\square$ the friction factor $\beta=4 r_{e}^{2} /[3 \ln (\xi)]$, which depends on the aggregate aspect ratio $r_{e}=L / A$. As already mentioned, the aggregates misaligned with the streamlines experience tensile hydrodynamic forces that tend to break them but, inside the aggregates, magnetic particles are subjected to magnetic attractive forces that consolidate the aggregates. Both hydrodynamic and magnetic forces depend on the aggregate orientation, so, they vary when the aggregate oscillates under the action of random torques. At some given orientation, hydrodynamic forces can overcome the magnetic ones and the aggregates will break. At other moments, the magnetic forces will dominate and the broken parts of the aggregates may join each other. Thus, the aggregates are expected to undergo a continuous fragmentation/aggregation process, and their aspect ratio should constantly fluctuate with the time around some mean value. Nevertheless, at this stage we neglect kinetics of this process and assume that all the aggregates have the same aspect ratio defined by the balance of the mean hydrodynamic and mean magnetic forces. In more details, the average aspect ratio of the aggregates is obtained as for the case of single chains [Martin and Anderson (1996)] supposing that the number of chains per unit area of the aggregate corresponds to a simple cubic packing of particles; in this case, the cohesive force can be estimated as: $F^{m}=(3 / 2) \Phi_{a} \pi A^{2} f^{m} \cos ^{2} \theta$. Here $\Phi_{a}=\pi / 6$ is the particle volume fraction inside the aggregates, $f^{m}$ is the magnetic force between two particles aligned with the external field per unit particle cross-section, $\pi a^{2}, a$ is the particle radius and $\theta$ is the angle between the magnetic field and the aggregate major axis; $\cos ^{2} \theta=h_{l} h_{s} e_{l} e_{s}$. The interparticle force per unit 
cross-section $f^{m}$ depends on the intensity of the external magnetic field as well as on particle magnetic properties. The hydrodynamic tensile force acting on aggregates is obtained by integration of the linear force density, $f_{\|}$[first expression in Eq. (5)], over the half of the aggregate length. Under the present assumptions, the balance of the mean magnetic and mean hydrodynamic forces reads:

$$
\frac{\pi L^{2}}{\ln (\xi)} \eta_{0} \gamma_{i k}\left\langle e_{i} e_{k}\right\rangle=\frac{3}{2} \pi A^{2} \Phi_{a} f^{m} h_{l} h_{s}\left\langle e_{l} e_{s}\right\rangle
$$

For the given geometry presented in Fig. 1, the components of the quantities $h_{i}, \gamma_{i k}, \omega_{i k}$ along the coordinates axes $\mathrm{O} x_{1}, \mathrm{O} x_{2}$ and $\mathrm{O} x_{3}$ (intervening into Eqs. (8), (9), (11)) take the values as follows: $h_{1}=1, h_{2}=h_{3}=0, \gamma_{12}=\gamma_{21}=\dot{\gamma} / 2, \omega_{12}=-\omega_{21}=\dot{\gamma} / 2, \gamma_{i k}=\omega_{i k}=0$ at $i k \neq 12,21$. Taking these relations into account and using the Eq. (11), we obtain the following expression for aggregate aspect ratio, or rather the form factor $\beta$ :

$$
\beta=\frac{2 \Phi_{a} f^{m}\left\langle e_{1}^{2}\right\rangle}{\eta_{0} \dot{\gamma}\left\langle e_{1} e_{2}\right\rangle}
$$

Substituting the last expression into the Eq. (7), we obtain that the rotational diffusion constant is linear in shear rate:

$$
D_{r}=\alpha\left(\frac{\left\langle e_{1} e_{2}\right\rangle}{\left\langle e_{1}^{2}\right\rangle} \frac{\Phi \mu_{0} \chi_{a}^{2} H^{2}}{2 \Phi_{a}^{2} f^{m}}\right)^{2} \dot{\gamma} \equiv C \dot{\gamma}
$$

Such shear rate dependence comes from the two opposite effects. First, the duration of angular jumps of aggregates scales as $\dot{\gamma}^{-1}$, so the diffusion constant must decrease inversely proportionally to the shear rate. Second, the aggregate length decreases with the shear rate. This decreases the viscous friction of aggregates proportionally to the shear rate and gives a 
quadratic growth of the diffusion constant with the shear rate. The product of both effects gives the linear dependence: $D_{r}=C \dot{\gamma}$. Note, that the same shear rate dependence was postulated by Folgar and Tucker (1984) for the rotational diffusion of non-Brownian rod-like particles induced by their collisions in sheared suspensions. However, the physics is quite different because, in the latter situation, the random orientional walk was only dictated by the rate of collisions proportional to the shear rate, whereas, in our case, it is the interplay between long range dipolar forces and shear rate, which produces the same scaling.

Substituting the Eqs. (9), (10), (12), (13) into Eq. (8) and considering the steady state regime with $d\left\langle e_{i} e_{k}\right\rangle / d t=0$, we obtain the system of equation for each of 9 components $\left\langle e_{i} e_{k}\right\rangle$ of the second statistical moment tensor. Because of the symmetry of the problem with respect to the coordinate plane $\mathrm{O} x_{1} x_{2}$ [Fig. 1], we pose $\left\langle e_{1} e_{3}\right\rangle=\left\langle e_{2} e_{3}\right\rangle=0$. Thus, taking into account the symmetry of this tensor, the number of unknown components reduces to four, namely, $\left\langle e_{1} e_{2}\right\rangle,\left\langle e_{1}^{2}\right\rangle,\left\langle e_{2}^{2}\right\rangle,\left\langle e_{3}^{2}\right\rangle$, and the three last of them are related by the expression $\left\langle e_{1}^{2}\right\rangle+\left\langle e_{2}^{2}\right\rangle+\left\langle e_{3}^{2}\right\rangle=1$, which comes from the fact that the norm of the unit vector $\mathbf{e}$ is equal to one. Thus, we obtain from the Eq. (8) the system of four algebraic equations, as follows:

$$
\left\{\begin{array}{l}
\left\langle e_{1} e_{2}\right\rangle-\left\langle e_{1}^{2}\right\rangle\left\langle e_{1} e_{2}\right\rangle+C_{2} \frac{\left\langle e_{1} e_{2}\right\rangle}{\left\langle e_{1}^{2}\right\rangle}\left(\left\langle e_{1}^{2}\right\rangle-\left\langle e_{1}^{2}\right\rangle^{2}\right)+C_{1}\left(\frac{\left\langle e_{1} e_{2}\right\rangle}{\left\langle e_{1}^{2}\right\rangle}\right)^{2}\left(1-3\left\langle e_{1}^{2}\right\rangle\right)=0 \\
-\left\langle e_{2}^{2}\right\rangle\left\langle e_{1} e_{2}\right\rangle-C_{2} \frac{\left\langle e_{1} e_{2}\right\rangle}{\left\langle e_{1}^{2}\right\rangle}\left\langle e_{1}^{2}\right\rangle\left\langle e_{2}^{2}\right\rangle+C_{1}\left(\frac{\left\langle e_{1} e_{2}\right\rangle}{\left\langle e_{1}^{2}\right\rangle}\right)^{2}\left(1-3\left\langle e_{2}^{2}\right\rangle\right)=0 \\
\left\langle e_{2}^{2}\right\rangle-2\left\langle e_{1} e_{2}\right\rangle^{2}+C_{2} \frac{\left\langle e_{1} e_{2}\right\rangle}{\left\langle e_{1}^{2}\right\rangle}\left(\left\langle e_{1} e_{2}\right\rangle-2\left\langle e_{1}^{2}\right\rangle\left\langle e_{1} e_{2}\right\rangle\right)-6 C_{1}\left(\frac{\left\langle e_{1} e_{2}\right\rangle}{\left\langle e_{1}^{2}\right\rangle}\right)^{2}\left\langle e_{1} e_{2}\right\rangle=0 \\
\left\langle e_{3}^{2}\right\rangle=1-\left\langle e_{1}^{2}\right\rangle-\left\langle e_{2}^{2}\right\rangle
\end{array}\right.
$$


where $\quad C_{1}=\alpha\left[\Phi \mu_{0} \chi_{a}{ }^{2} H^{2} /\left(2 \Phi_{a}{ }^{2} f^{m}\right)\right]^{2} \quad$ and $\quad C_{2}=\mu_{0} \chi_{a}{ }^{2} H^{2} /\left[2 \Phi_{a} f^{m}\left(2+\chi_{a}\right)\right] \quad$ are dimensionless factors independent of shear rate. Note that, because of the relations $D_{r}=C \dot{\gamma}$ and $\beta \propto \dot{\gamma}^{-1}$, all the terms on the right-hand side of the Eq. (8) become linear with the shear rate. Therefore, in the steady state regime, the shear rate disappears from the Eqs. (8), (14), so, the aggregate orientations are independent of shear rate. Analysis shows that, in our experiments, the parameters $C_{1}$ and $C_{2}$ take the values $0.05<C_{1}<1$ and $0.04<C_{2}<0.12$. So, in general case the system (14) is solved numerically. However, in the wide range of magnetic fields and concentrations $(H<15 \mathrm{kA} / \mathrm{m}$ and $\Phi<0.3)$, the parameter $C_{1}$ remains relatively small $\left(C_{1}<0.2\right)$ and the system (14) admits, within the $10 \%$ error, an approximate analytical solution, as follows:

$\left\langle e_{1} e_{2}\right\rangle=\frac{C_{1}}{\left(1+C_{2}\right)\left(2+C_{2}\right)}, \quad\left\langle e_{2}^{2}\right\rangle=\left\langle e_{3}^{2}\right\rangle=\frac{C_{1}^{2}}{\left(1+C_{2}\right)^{2}\left(2+C_{2}\right)}, \quad\left\langle e_{1}^{2}\right\rangle=1-\frac{2 C_{1}^{2}}{\left(1+C_{2}\right)^{2}\left(2+C_{2}\right)}$

The diagonal components $\left\langle e_{i}^{2}\right\rangle$ of the second statistical moment can be seen as the mean square cosines of the angle between the aggregate major axis and the O $x_{i}$ coordinate axis. For example, $\left\langle e_{1}^{2}\right\rangle$ is the mean square cosine of the angle that the aggregate makes with the flow. At small magnetic fields and particle concentrations, the $\left\langle e_{1} e_{2}\right\rangle$ component can be interpreted as a mean sine of the angle between the aggregates and the flow.

Let us now estimate the shear stress developed in the suspension. The most general expression for the stress tensor of a dilute suspension composed of axisymmetric aggregates subject to both an external torque (magnetic torque $\mathbf{T}^{m}$ in our case) and rotational diffusion is given by Pokrovskiy (1978). In more concentrated suspensions, long-range hydrodynamic interactions between aggregates will enhance viscous dissipation but we can still use the expression 
derived for the dilute suspensions upon replacing the form factor $\beta$ by the appropriate value, $\beta=4 r_{e}^{2} /[3 \ln (\xi)]$. A similar trick has been used by Bird et al. (1977) when computing the stress in a semi-dilute solution of rod-like polymer. For the suspension of slender aggregates the expression for the stress tensor reduces to:

$$
\begin{gathered}
\sigma_{i k}=-p \delta_{i k}+2 \eta_{0} \gamma_{i k}+\frac{\Phi}{\Phi_{a}} \eta_{0}\left\{4 \gamma_{i k}+\frac{\beta}{2}\left[\left\langle e_{i} e_{k} e_{l} e_{m}\right\rangle-\frac{1}{3} \delta_{i k}\left\langle e_{l} e_{m}\right\rangle\right] \gamma_{l m}\right\}+ \\
+\frac{\Phi}{\Phi_{a} V_{a}}\left\{\frac{1}{2}\left[\left\langle e_{i} e_{l} T^{m}{ }_{l k}\right\rangle+\left\langle e_{k} e_{l} T^{m}{ }_{l i}\right\rangle\right]-\frac{1}{2}\left\langle T^{m}{ }_{i k}\right\rangle\right\}+\frac{\Phi}{\Phi_{a}} \beta \eta_{0} D_{r}\left[3\left\langle e_{i} e_{k}\right\rangle-\delta_{i k}\right]
\end{gathered}
$$

where $p$ is the pressure. Replacing the magnetic moment $T^{m}{ }_{i k}$ by the Eq. (9) and taking into account the Eqs. (10), (12), (13) for $\left\langle e_{i} e_{k} e_{l} e_{m}\right\rangle, \beta$ and $D_{r}$, we obtain the following expression for the shear stress $\sigma_{12}$ :

$$
\begin{aligned}
\sigma_{12}= & \underbrace{\eta_{0} \dot{\gamma}\left(1+2 \frac{\Phi}{\Phi_{a}}\right)}_{\text {solvent stress }}+\underbrace{\Phi f^{m}\left\langle e_{1} e_{2}\right\rangle\left\langle e_{1}^{2}\right\rangle}_{\begin{array}{c}
\text { hydrodynamic } \\
\text { aggregate stress }
\end{array}} \underbrace{-2 \frac{\Phi}{\Phi_{a}} \cdot \frac{\chi_{a}^{2}}{2+\chi_{a}} \mu_{0} H^{2}\left\langle e_{1} e_{2}\right\rangle\left\langle e_{2}^{2}\right\rangle}_{\text {magnetic stress }}+ \\
& +\underbrace{\frac{3}{2} \alpha \frac{\Phi^{3}}{\Phi_{a}^{4}} \frac{\left(\mu_{0} \chi_{a}^{2} H^{2}\right)^{2}}{f^{m}} \frac{\left\langle e_{1} e_{2}\right\rangle^{2}}{\left\langle e_{1}^{2}\right\rangle}}_{\text {diffuision stress }}
\end{aligned}
$$

The first term in the right-hand side of this equation stands for the solvent contribution to the stress and the last three terms stand for the aggregate contribution. Among these three terms, the first one corresponds the hydrodynamic part of the aggregate stress, the second one comes from the external magnetic torque (magnetic stress) and the last one arises from the random interaction torques inducing random fluctuations of aggregate orientations (defined by Leal and Hinch (1972) as diffusion stress). All the three contributions of the aggregate stress appear to be independent of the shear rate, so, their sum is considered as a dynamic yield 
stress. Furthermore, analysis shows that the magnetic stress gives a negligible contribution, so the final expression for the yield stress reads:

$$
\sigma_{Y} \approx \underbrace{\Phi f^{m}\left\langle e_{1} e_{2}\right\rangle\left\langle e_{1}^{2}\right\rangle}_{\text {hydrodynamic stress }}+\underbrace{\frac{3}{2} \alpha \frac{\Phi^{3}}{\Phi_{a}{ }^{3}} \frac{\left(\mu_{0} \chi_{a}^{2} H^{2}\right)^{2}}{f^{m}} \frac{\left\langle e_{1} e_{2}\right\rangle^{2}}{\left\langle e_{1}^{2}\right\rangle}}_{\text {diffusion stress }}
$$

As expected, this stress appears to be independent of shear rate due to the fact that the hydrodynamic stress is proportional to $\eta_{0} \dot{\gamma} r_{e}^{2}$ and the diffusion stress is proportional to $\eta_{0} r_{e}^{2} D_{r}$ with $r_{e}^{2} \propto \dot{\gamma}^{-1}$ and $D_{r}=C \dot{\gamma}$. In conclusion, our theory predicts a linear Bingham-like rheological law, $\sigma_{12}=\sigma_{Y}+\eta \dot{\gamma}$, for the magnetic suspension in the presence of longitudinal magnetic field. The plastic viscosity, $\eta=\eta_{0}\left(1+2 \Phi / \Phi_{a}\right)$ appears to be field-independent and proportional to the solvent viscosity, $\eta_{0}$. This conclusion is, however, valid for the aggregates long enough, for which the slender body approach, used in our theory, remains valid. This corresponds to the form factor $\beta \sim r_{e}^{2}{ }_{\leftrightarrow} 100$, or, with the help of the Eq. (12), we obtain: $\eta_{0} \dot{\gamma} / f_{m} \bigcirc\left[100\left\langle e_{1} e_{2}\right\rangle\right]^{-1}$. We have checked that this condition is satisfied in our experiments.

For the quantitative estimations of the shear stress we need to estimate the magnetic susceptibility of the aggregates, $\chi_{a}$ and the magnetic force $f^{m}$ per unite cross-section of particles composing the aggregates. We should keep in mind that closely spaced magnetic particles inside the aggregates mutually bias each other and thus cannot be considered as point dipoles. It has been shown that the dipolar approach underestimates the interparticle forces by one or two orders of magnitudes [Clercx and Bossis (1993), Ginder and Davis (1994)]. It also gives understated values of the magnetic susceptibility of aggregates [Clercx and Bossis (1993)]. Therefore, in order to predict at least a correct order of magnitude for the yield stress, 
we must use more precise approaches for calculations of $f^{m}$ and $\chi_{a}$. In more details, we estimate both quantities for a simple cubic arrangement of particles in the aggregates by calculating first the magnetic field distribution in the aggregates aligned with the magnetic field. After that, we derive the interparticle force by integrating the Maxwell stress tensor over the particle surface and, finally, we estimate the magnetic susceptibility of aggregates as $\chi_{a}=\left\langle M_{a}\right\rangle /\left\langle H_{a}\right\rangle$, where $\left\langle M_{a}\right\rangle$ and $\left\langle H_{a}\right\rangle$ are, respectively, the magnetization and the magnetic field intensity in the aggregate, both averaged over the aggregate volume. The numerical simulations are carried out using finite element method and a more detailed description of the calculation procedure can be found in [Ginder and Davis (1994)]. In our simulations, we suppose that the particle surfaces are separated from each other by a small nonmagnetic gap of $4 \mathrm{~nm}$ (about 0.0013 of the particle diameter), which corresponds to the double thickness of the surfactant layer on the particle surface. Actually, at such small separation, the interparticle force is only a few percent smaller than the one in the case of a close contact of particles. We also use a Fröhlich-Kennelly non-linear magnetization law for the carbonyl iron magnetic particles [Jiles (1991)]: $M_{p}=\chi_{i} M_{S} H_{p} /\left(M_{S}+\chi_{i} H_{p}\right)$ with $M_{p}$ and $H_{p}$ being the magnetization and the magnetic field intensity inside the particles, $\chi_{i} \approx 130$ and $M_{S} \approx 1360 \mathrm{kA} / \mathrm{m} \square$ the initial magnetic susceptibility and saturation magnetization of particles [Bossis et al. (2002-a)]. The calculated quantities $f^{m}$ and $\chi_{a}$ are functions of the magnetic field intensity and, in the range of magnetic fields, $2<H<20 \mathrm{kA} / \mathrm{m}$, are approximated by the following polynomials:

$$
f^{m}(H)=\left(-1.34 \cdot 10^{5}\left(\frac{H}{M_{S}}\right)^{2}-3.62 \cdot 10^{3} \frac{H}{M_{S}}+162\right) \mu_{0} H^{2}
$$




$$
\chi_{a}(H)=-2.77 \cdot 10^{3}\left(\frac{H}{M_{S}}\right)^{2}-23.4 \frac{H}{M_{S}}+9.22
$$

To validate our theory, we perform a detailed experimental study of magnetic suspension flow in the presence of a longitudinal magnetic field. In the following Section III, we present experimental techniques as well as data treatment methods.

\section{Experimental}

Experimental realization of simple shear flows with the magnetic field aligned with the fluid streamlines remains problematic. The use of sliding-plate rheometers is limited by technical problems with realization of steady-state flows [Macosko (1994)]. In standard rotational rheometers, circular flows are induced between rotating disks or concentric cylinders, but it is not trivial to create a circular homogeneous magnetic field. A non-homogeneous circular field created by an electric current of a cylindrical conductor would cause a substantial migration of magnetic particles towards the conductor. These technical problems push us to use a pressure-driven flow through a cylindrical channel instead of the simple shear flow studied theoretically. The velocity profile is not linear in capillary flows, so the shear rate varies from zero at the channel axis to a maximum value at the wall. However, using our model, we estimate that the shear rate variation, $\Delta \dot{\gamma}$, along the aggregates is negligible, except for a narrow central flow region: $\Delta \dot{\gamma}(r) / \dot{\gamma}(r) \approx L \sin \theta / r \sim(a / r) \cdot\left[f_{m}\left\langle e_{1} e_{2}\right\rangle /\left(\eta_{0} \dot{\gamma}\right)\right]^{1 / 2} \ll 1$ for $r / R_{\leftrightarrow} 0.1$, with $r$ being radial coordinate, $R \square$ channel radius, $a \square$ particle radius and $\theta$ - the angle between the channel axis and the aggregate $s$ major axis [Fig. 1]. In the last expression, we replaced $\sin \theta$ by $\left\langle e_{1} e_{2}\right\rangle$ and estimated the aggregate length as $L \sim a \cdot \beta^{1 / 2}$ with $\beta$ given by the Eq. (12). So, we expect that the rheological behavior observed in the pressure-driven flow should be similar to that in a 
drag shear flow with linear velocity profile. We also expect that our theory, developed for linear shear flows, can be applied safely for capillary flows, at least, within the range of experimental parameters used in the present work.

Pressure-driven flow through cylindrical channels was realized using the capillary rheometer Rosand RH7 (Malvern Instruments). The apparatus allows applying a flow rate, $Q$, in a stepwise manner and the pressure difference, $\Delta p$, between both extremities of the channel, is measured by a strain gauge pressure transducer. The duration of the flow-rate steps was sufficiently long to achieve a stable steady-state pressure response, at which fluctuations in $\Delta p$ did not exceed two per cent of the mean value. Flow channels, of two different diameters $(0.5$ and $0.75 \mathrm{~mm})$ and five different lengths $(0.25,0.5,1,2$ and $4 \mathrm{~mm})$, were fabricated from aluminum cylinders by micro-drilling. A homogeneous magnetic field of an intensity, $H_{0}$, ranging from 0 to $15 \mathrm{kA} / \mathrm{m}$ and parallel to the channel axis, was generated by a pair of Helmholtz coils placed co-axially around the channel. Once, the pressure-flow rate curves are measured, we determine the shear stress versus shear rate dependences $\square$ flow curves $\square$ by using the standard procedure [Macosco (1994)]. Bagley and Mooney-Rabinowitch corrections were applied for calculations of the flow curves in order to exclude the effects of entry and exit flows and to correct the shear rate for non-Newtonian flows. Possible effects of wall slip are checked by using the channels of two different diameters. The coincidence of the flow curves for the channels of both diameters in the whole range of experimental parameters allows us to not consider this effect [Macosco (1994)].

The MR fluids used in this work were composed of spherical iron particles (AnalaR Normapur; Prolabo®; VWR International) of mean diameter of $2 a=3 \mu \mathrm{m}$ and magnetization saturation, $M_{S}=1360 \mathrm{kA} / \mathrm{m}$. These particles were dispersed in a silicon oil (Rhodorsil ${ }^{\circledR}$; VWR International; dynamic viscosity at $25^{\circ} \mathrm{C}$ is $\eta_{0}=0.479 \mathrm{~Pa} \cdot \mathrm{s}$ ) at three volume fractions, $\Phi=0.2$, 
0.3 and 0.4 , and stabilized against aggregation by an appropriate amount of aluminum stearate (Sigma-Aldrich).

In the capillary rheometer, the MR fluid is pushed from a large cylindrical container into a narrow capillary coaxial with respect to the container. Since the magnetic field lines are partially $\lceil$ confined $\square$ within the flow pass of the MR fluid, the mean magnetic flux density in the MR fluid inside the capillary will be higher than that inside the container. We have performed numerical simulations of the magnetic field distribution in the experimental geometry and found that the mean magnetic field intensity $H$ inside the capillary is about 1.5 times the intensity $H_{0}$ of the external magnetic field created by the Helmholtz coils. Nevertheless, the magnetic field inside the capillary varied around the mean value $H$ only by a few per cent and was therefore considered as uniform. The main variation of the magnetic field occurred in the container in the vicinity of the capillary entrance, so, the field gradients may affect the entrance pressure loss. However, this should not influence the results for the shear stress in the capillary because we subtract the entrance pressure loss from the total measured pressure difference $\Delta p$ performing the Bagley correction. The entrance flows of MR suspensions have been considered in details by Kuzhir et al. (2009). In what follows, we analyze the field effects on the aggregate orientation and on the suspension stress with regard to the mean magnetic field inside the capillary, $H \approx 1.5 H_{0}$.

\section{Results and discussion}

The experimental flow curves obtained for the capillary flow in the presence of a longitudinal magnetic field are shown in Fig. 2 for the particle volume fraction $\Phi=0.3$. As is seen from this figure, the flow curves, obtained for different magnetic field intensities are linear and parallel to each other within the whole experimental range of shear rates, $500<\dot{\gamma}^{-1}<3000 \mathrm{~s}^{-1}$. The 
increase in the magnetic field intensity causes an increase in the stress level of the suspension such that the flow curves are shifted upwards but their slopes remain the same. So, these curves can be interpolated by a linear rheological law, $\sigma_{12}=\sigma_{Y}+\eta \dot{\gamma}$ with $\sigma_{Y}$ being a fielddependent dynamic yield stress (an intercept of extrapolated curves onto zero shear rate) and $\eta \square \mathrm{a}$ field independent plastic viscosity. Note that our theory predicts the similar Bingham like behavior with field-independent plastic viscosity [Eq. (17)]. Therefore, both in theory and in experiments, the dynamic yield stress of the suspension appears to be the major rheological property influenced by a magnetic field. In what follows, we shall concentrate our attention on this particular property.

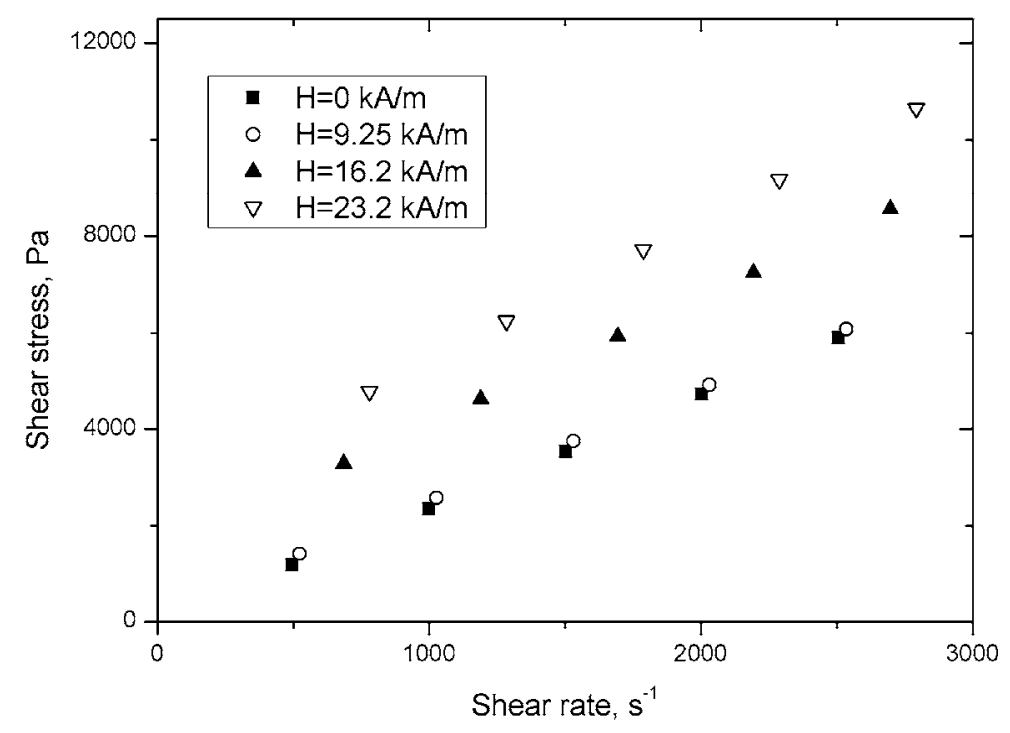

Fig. 2. Experimental flow curves of the MR fluid in the presence of an external longitudinal magnetic field of different intensities. These curves were obtained for a pressure-driven flow using the capillary rheometer. The particle volume fraction of the suspension was $\Phi=0.3$.

Experimental and theoretical dependencies of the dynamic yield stress on the magnetic field intensity are presented in Fig.3 for the magnetic suspensions of different volume fractions. The theoretical dependencies were fitted to experimental ones by using the least square method with a single free parameter, $\alpha$ - interaction constant intervening into the Eq. (13) for the diffusion constant. Interestingly, the three presented curves for three different volume 
fractions of the MR fluid are fitted reasonably well by a single value of the interaction constant $\alpha=1.5$. Possible reasons for a relatively small discrepancy between theoretical and experimental curves at $\Phi=0.4$ and $H>18 \mathrm{kA} / \mathrm{m}$ (upper curve in Fig.3) are discussed below.

As is seen from Fig. 3, the yield stress is an increasing function of both magnetic field intensity and the suspension volume fraction. The increasing field-dependence of the yield stress can be easily understood by the two mechanisms, as follows. First, the magnetic interactions between aggregates increase with the increasing magnetic field. This leads to larger fluctuations of aggregate orientation and therefore to a larger viscous dissipation. This mechanism appears in the Eq. (18) for the yield stress through the statistical moment $\left\langle e_{1} e_{2}\right\rangle$, which should be a growing function of the magnetic field intensity as will be discussed below [cf. Fig. 6c]. Second, magnetic interactions between particles, composing the aggregates, also increase with a growing magnetic field. The aggregates become more resistive against destructive shear forces, their length increases with the field, so, they generate higher stresses. In more details, the hydrodynamic stress on aggregates is proportional to the aggregate aspect ratio squared, $r_{e}^{2}$, and the later is proportional to the interparticle magnetic force per unit particle cross-section, $f^{m}$, which is increasing function of the magnetic field intensity. Finally, these two mechanisms contribute simultaneously into the increasing field-response of the yield stress. 


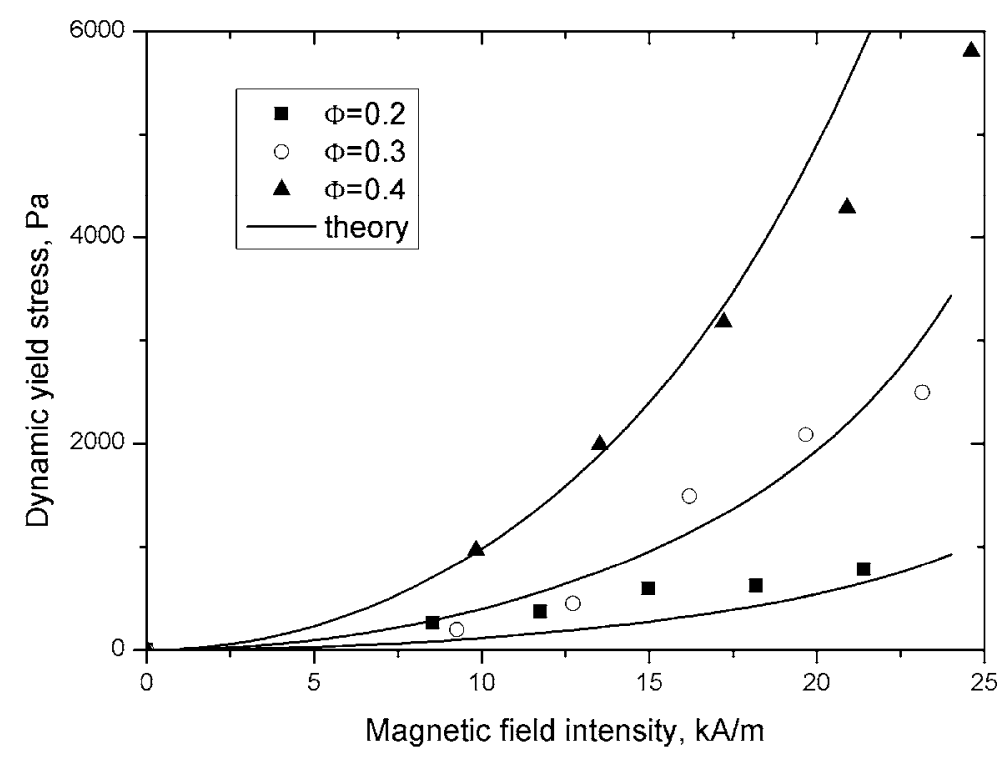

Fig. 3. Theoretical and experimental dependencies of the dynamic yield stress of the MR fluids on the magnetic field strength at different particle volume fractions. Points correspond to experimental data and lines $\square$ to the theory.

The concentration dependence of the dynamic yield stress is presented in Fig. 4 for the magnetic field strength $H=15 \mathrm{kA} / \mathrm{m}$. The theoretical values of the yield stress were calculated using the appropriate value of the free parameter, $\alpha=1.5$ found previously by fitting theoretical field dependencies of the yield stress to the experimental ones. Again, we obtain a reasonably good correspondence with experiments. As is seen from Fig. 4, both theory and experiments show that the yield stress increases with the particle volume fraction stronger than linearly. Such nonlinear behavior could be easily explained by concentration-enhanced interactions between aggregates. In more details, the mean distance between aggregates and, consequently, the magnetic interaction torque increase with the particle volume fraction. Therefore, the aggregates will be subjected to stronger fluctuations of their orientation and will generate a stronger viscous dissipation. 


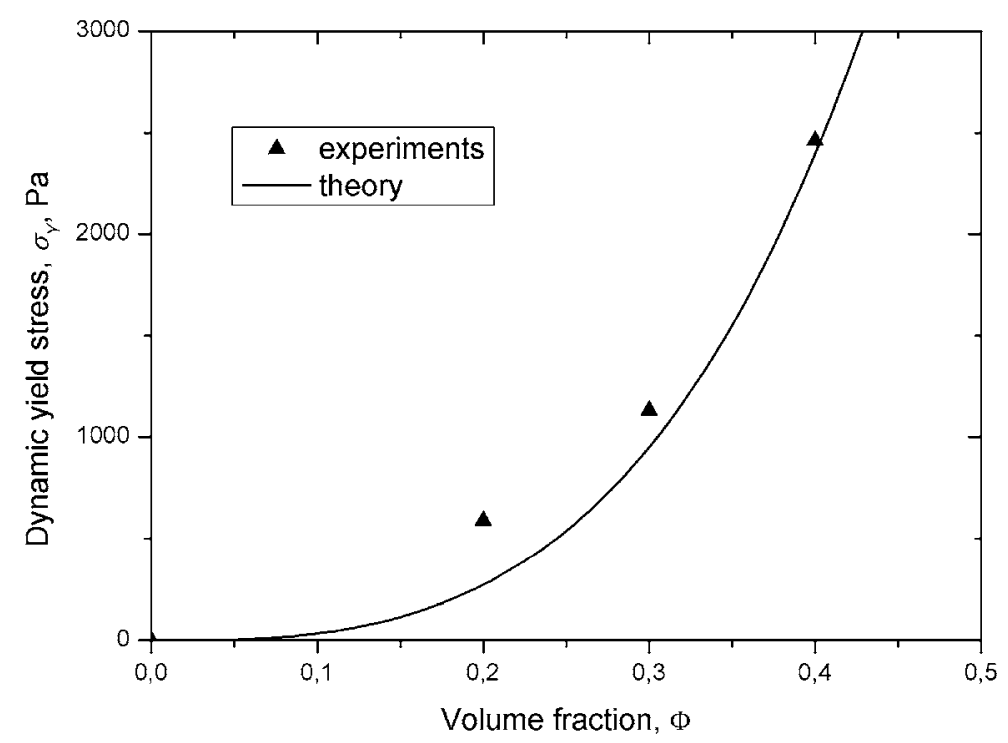

Fig.4. Theoretical and experimental dependencies of the dynamic yield stress on the particle volume fraction for the magnetic field strength $H=15 \mathrm{kA} / \mathrm{m}$.

For a more quantitative analysis of the field and concentration effects on the yield stress, let us inspect these effects on two separate components of the computed yield stress hydrodynamic aggregate stress and diffusion stress. The magnetic stress appears to be negligible in our model and is not worth attention. The concentration dependence of both components of the stress is shown in Fig. 5a for the magnetic field intensity, $H=15 \mathrm{kA} / \mathrm{m}$. As is seen in this figure, the hydrodynamic stress dominates over the diffusion stress, however, the later shows a stronger concentration behavior. For example, at the volume fraction $\Phi=0.3$, the relative contributions of the diffusion and hydrodynamic terms to the total yield stress are 12 and 88 percents, respectively, but at the volume fraction $\Phi=0.4$, these contributions are 32 and 68 percents. Thus, the relative importance of the diffusion stress increases with the particle concentration. This can be easily understood by analyzing the expression (18) for the yield stress. According to this equation, the hydrodynamic stress $\sigma_{H}$ is proportional to $\Phi\left\langle e_{1} e_{2}\right\rangle\left\langle e_{1}^{2}\right\rangle$ and the diffusion stress $\sigma_{D}$ is proportional to $\Phi^{3}\left\langle e_{1} e_{2}\right\rangle^{2} /\left\langle e_{1}^{2}\right\rangle$. At relatively low concentrations, when the approximate expressions (15) hold, $\left\langle e_{1}^{2}\right\rangle \sim 1$ and $\left\langle e_{1} e_{2}\right\rangle \sim C_{1} \propto \Phi^{2}$, 
therefore, the hydrodynamic stress varies as $\sigma_{H} \propto \Phi^{3}$ and the diffusion stress shows even stronger power-law dependence on concentration: $\sigma_{D} \propto \Phi^{7}$. In fact, the component $\left\langle e_{1} e_{2}\right\rangle$ of the second statistical moment can be roughly seen as the mean angle $\theta$ between the aggregate and the flow [Fig. 1]. With the growing concentration, the magnetic interactions between the aggregates become stronger, and this leads to stronger deviations of the aggregate orientation from the flow direction, hence, to higher values of the mean angle $\theta \approx\left\langle e_{1} e_{2}\right\rangle \propto \Phi^{2}$ and to higher hydrodynamic stresses. An unexpectedly strong concentration effect on the diffusion stress can be interpreted in terms of the concentration behavior of the diffusion constant. According to the Eq. (13), $D_{r} \propto\left(\Phi\left\langle e_{1} e_{2}\right\rangle\right)^{2} \propto \Phi^{6}$. This behavior shown in Fig. $5 \mathrm{~b}$ comes from the two effects as follows. On the one hand, the diffusion is enhanced by the magnetic interactions between aggregates, which become stronger with the growth in concentration, such that $D_{r} \propto\left\langle T_{\text {int }}{ }^{2}\right\rangle \propto \Phi^{2}$ [cf. Eqs. (2), (4)]. On the other hand, with increasing orientation fluctuations, the destructive shear forces increase and the mean aggregate length decreases, which facilitates the diffusion process and $D_{r} \propto f_{r}^{-2} \propto \beta^{-2} \propto\left\langle e_{1} e_{2}\right\rangle^{2} \propto \Phi^{4}$ [cf. Eqs. (2), (7), (12)]. Both these effects amplify each other and lead to the final dependence $D_{r} \propto \Phi^{6}$. 

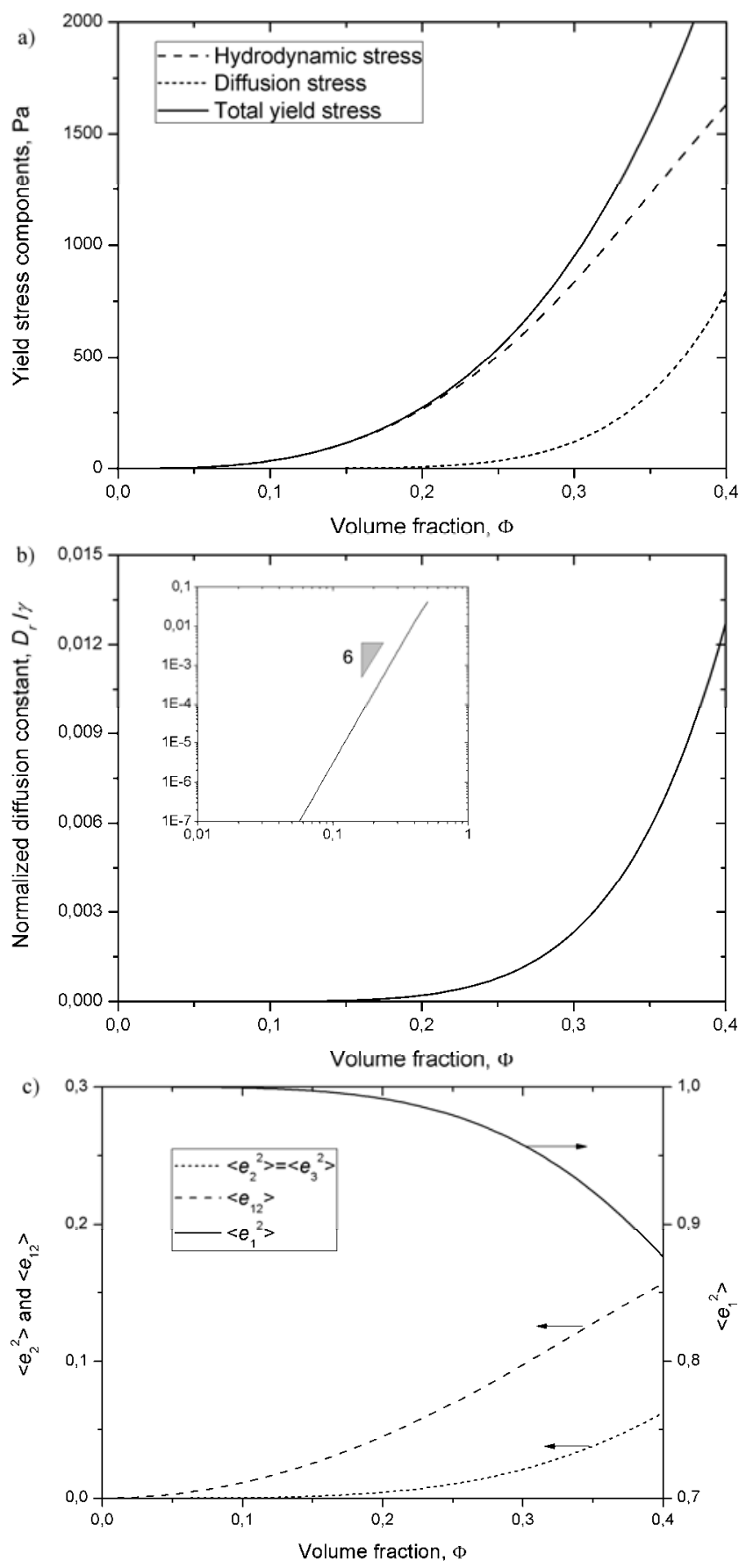

Fig. 5. Theoretical concentration-dependencies of the hydrodynamic and diffusion components of the yield stress (a), of the diffusion constant normalized by the shear rate $\dot{\gamma}$ (b) and of the components of the second statistical moment (c). The magnetic field intensity is $\mathrm{H}=15 \mathrm{kA} / \mathrm{m}$. The $\log$-log scale of the $D_{r}$ versus $\Phi$ dependence allows one to visualize the $\Phi^{6}$ law for the rotary diffusivity (inset of the figure (b)).

Note that we constructed our model assuming relatively small angular fluctuations, such that the space, within which an aggregate oscillates, does not overlap the $\llbracket$ space of activity $\square$ of any other aggregate. Quantitatively, this restriction corresponds to the condition 
$\left\langle e_{1} e_{2}\right\rangle \bigcirc d / L \sim(A / L)\left(\Phi / \Phi_{a}\right)^{-1 / 2}$ with $d$ being the mean distance between aggregates [cf. Fig.1]. The aggregate aspect ratio is estimated using the Eq. (12): $r_{e}=L / A \sim \beta^{1 / 2} \sim\left[f^{m} /\left(\eta_{0} \dot{\gamma}\left\langle e_{1} e_{2}\right\rangle\right)\right]^{1 / 2}$. So, the condition, at which the angular fluctuations of aggregates are not restricted by the presence of neighboring aggregates, takes the final form, as follows: $\eta_{0} \dot{\gamma} / f^{m}{ }_{\leftrightarrow} \Phi\left\langle e_{1} e_{2}\right\rangle$. Together with the restriction on aggregate length [cf. discussion below Eq. (18)], the range of validity of our model becomes: $\Phi\left\langle e_{1} e_{2}\right\rangle \bigcirc \eta_{0} \dot{\gamma} / f^{m} \bigcirc\left[100\left\langle e_{1} e_{2}\right\rangle\right]^{-1}$. Estimations show that this condition is verified for the concentrations $\Phi \odot 0.3$ in the whole range of the experimental shear rates and magnetic fields. For higher concentration, $\Phi=0.4$, this condition is satisfied at $H<12 \mathrm{kA} / \mathrm{m}$ for any shear rate (in estimations, zero-field suspension viscosity was used for $\eta_{0}$ ). At higher magnetic fields and concentrations, the angular fluctuations will be hindered by direct contacts between aggregates. This effect should weaken the diffusion process. For instance, the diffusion constant of rod-like polymers decreases by two orders of magnitude between the dilute and concentrated regimes. Doi and Edwards (1986) proposed a tube model, which accounts for the topological constraints to the angular displacement of rod-like polymers through an effective diffusion constant, $D_{r}=D_{r} /\left[\Phi(L / A)^{2}\langle\sin \phi\rangle\right]^{2}$, where $\langle\sin \phi\rangle$ is the mean sine of the angle between the axes of two neighboring particles. Applying this model to our case, we find that, at high concentrations and magnetic fields, the effective diffusion constant becomes independent of concentration. This means that the effect of the mechanical constraint cancels completely both above mentioned effects leading to $\Phi^{6}$-behavior of $D_{r}$. This could maybe explain a discrepancy between the experimental and the theoretical field dependences of the yield stress at $\Phi=0.4$ and $H>18 \mathrm{kA} / \mathrm{m}$ [upper curve in Fig. 3]. According to the above 
mentioned estimations, at this concentration and magnetic fields, the aggregates are expected to collide with each other that would decrease the intensity of diffusion and generate a lower stress than the one predicted by our model. However, collisions between aggregates would significantly enhance the short-range hydrodynamic interactions and generate a supplementary stress, at least, proportional to $\Phi^{2}$ [Férec et al. (2009)]. Furthermore, a simple interpolation of Doi and Edward s theory to magnetic non-Brownian suspensions is not obvious. First, the rotational diffusion of aggregates will be mostly governed by collisions between them and by short-range hydrodynamics [Folgar and Tucker (1984)] rather than by the mechanism proposed by Doi and Edwards (1986). Second, colliding aggregates may break in collision points because of high concentration of hydrodynamic stress at these points. Thus, the aggregates are expected to constantly break during collisions and reform between collisions that could randomize their orientation. Because of complexity of the problem, we are not able, at the present time, to indicate concentration behaviors of the diffusion constant and of the suspension stress in high concentration/field limit. This question requires a detailed numerical study and will be considered in future.

Finally, we present the concentration dependencies of each component of the statistical moment $\left\langle e_{i} e_{k}\right\rangle$ in Fig. $5 \mathrm{c}$ for the magnetic field intensity $H=15 \mathrm{kA} / \mathrm{m}$. We see that the $\left\langle e_{1}^{2}\right\rangle-$ component (mean square cosine of the angle between the aggregate and the flow) decreases gradually from unity at low concentrations to 0.88 at $\Phi=0.4$. This means that the aggregates become less aligned with the flow with growing concentrations. Both $\left\langle e_{2}{ }^{2}\right\rangle$ and $\left\langle e_{1} e_{2}\right\rangle$ components increase with the particle concentration, and the $\left\langle e_{1} e_{2}\right\rangle$ component (mean sine of the angle between the aggregates and the flow) is always larger than the $\left\langle e_{2}{ }^{2}\right\rangle$-component (mean square cosine between the aggregate and velocity gradient axis Ox 2 [Fig. 1]). The 
interpolation of these curves to higher concentrations would give more or less isotropic orientations of aggregates. However, keep in mind, that the present model cannot be applied safely at $\Phi>0.4$ for the considered magnetic field. As already mentioned, at higher concentrations, the diffusion is hindered and we expect only a moderate variation of $\left\langle e_{1} e_{2}\right\rangle$, $\left\langle e_{1}^{2}\right\rangle$ and $\left\langle e_{2}^{2}\right\rangle$ with $\Phi$.

Let us inspect now the magnetic field effect on the two components of the yield stress, as well as on the diffusion constant and on the components of the second statistical moment. These dependencies are shown in Figs. 6a, b and c for the particle volume fraction $\Phi=0.3$. Similarly to the concentration behavior, the hydrodynamic stress dominates over the diffusion stress and the latter increases faster with the magnetic field intensity than the former. The field behavior of the hydrodynamic stress is well fitted by a second power-law, $\sigma_{H} \propto H^{2}$ while the diffusion stress is fitted by a fourth-order polynomial of $H$. Such non-quadratic behavior of the diffusion stress comes from the non-linear magnetization of magnetic particles composing the MR suspension. If the particles were linearly magnetized (had a constant magnetic permeability), the aggregate susceptibility $\chi_{a}$, the diffusion constant $D_{r}$ and the aggregate orientations $\left\langle e_{i} e_{k}\right\rangle$ would be field independent, and the interparticle magnetic force $f^{m}$ and both components of the yield stress would scale as $\mu_{0} H^{2}$, as follows from Eqs. (13), (14), (18)-(20). In the present case of non-linear magnetization, the neighboring aggregates bias each other to a lesser extent than the particles inside the aggregates. Therefore, the interparticle magnetic force saturates faster than the interaction torque between aggregates as the magnetic field increases. This leads to an increasing field dependence of the interaction torque $\square$ to interparticle force ratio, $\Omega \equiv \mu_{0} \chi_{a}^{2} H^{2} / f^{m}$. Since the diffusion constant varies as $\Omega^{2}$, it also increases with the magnetic field strength [Fig. 6b] and results in a strong field 
behavior of the diffusion stress. Similarly to the diffusion constant, the statistical moments are defined by the ratio $\Omega$. In highly aligned state of aggregate orientation, we get from the Eq. (15): $\left\langle e_{1} e_{2}\right\rangle \propto \Omega^{2}$ and $\left\langle e_{2}^{2}\right\rangle \propto \Omega^{4}$. This explains a stronger field dependence of the moment $\left\langle e_{2}^{2}\right\rangle$ as compared to $\left\langle e_{1} e_{2}\right\rangle$ [Fig. 6c]. Consequently, the mean-square cosine of the angle between the aggregate and the flow is a strongly decreasing function of the magnetic field $\left\langle e_{1}^{2}\right\rangle \approx 1-\lambda \Omega^{4}$, with $\lambda$ being a constant. Thus, at strong enough magnetic fields, we could expect an isotropic orientation of the aggregates. However, the interpolation of the field dependencies of the statistical moments to higher magnetic fields $(H>25 \mathrm{kA} / \mathrm{m}$ for $\Phi=0.3)$ is not correct because of collisions between aggregates and their possible collision-induced destruction. 

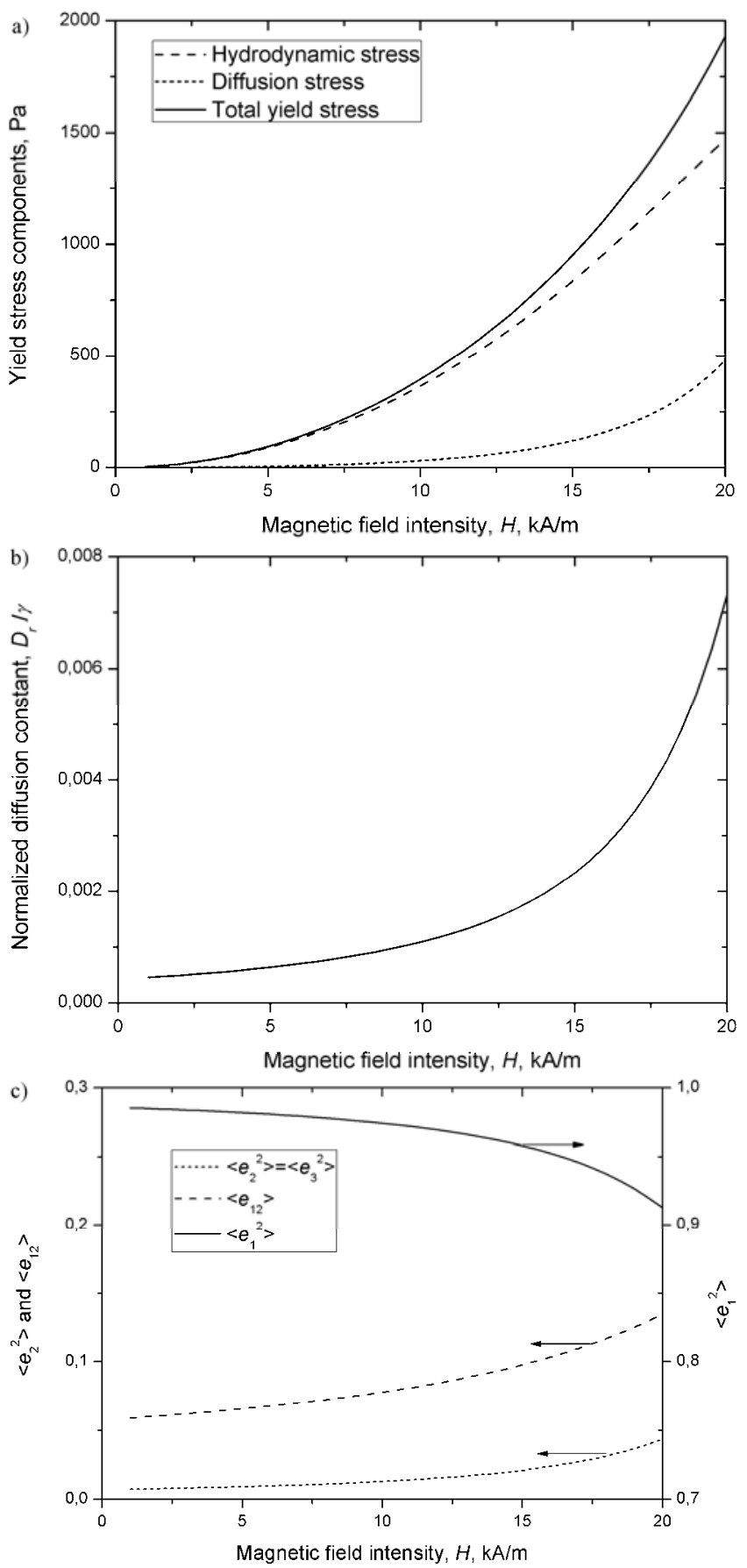

Fig. 6. Theoretical field-dependencies of the hydrodynamic and diffusion components of the yield stress (a), of the diffusion constant normalized by the shear rate $\dot{\gamma}$ (b) and of the components of the second statistical moment (c). The particle volume fraction is $\Phi=0.3$

It is worth to mention that, whatever the case, the mean sine of the angle between the aggregates and the flow $\left\langle e_{1} e_{2}\right\rangle$ is positive, meaning that the aggregates spend more time in the first quarter of the shear plane $\mathrm{O} x_{1} x_{2}$ [Fig. 1]. In fact, the shear flow tends to rotate the 
aggregates along the vorticity, in the clockwise direction, while the stochastic interaction torque tends to randomize their orientation and causes their back-diffusion against the vorticity, in the counterclockwise direction. This leads to an asymmetry about the flow direction in aggregate orientation distribution. Similar effect has been observed experimentally in shear flows of semi-diluted fiber suspensions [Stover et al. (1992)]. The asymmetry of fiber orientations with respect to the streamlines was attributed to collisions between fibers, which were accounted for by an effective rotary diffusive process [Folgar and Tucker (1984)].

Finally note that our theory does not cover possible solid-like behaviour of the MR fluid at zero shear rates. Prediction of the static yield stress (threshold stress required for onset of flow) is out of scope of the model. As mentioned above, the theory is valid for shear rates high enough $\left(\eta_{0} \dot{\gamma} / f^{m}{ }_{\leftrightarrow} \Phi\left\langle e_{1} e_{2}\right\rangle\right)$ such that the aggregates are relatively short and assumed to not interact with the walls neither collide with each other. Under such condition, the shear stress in the suspension is mainly defined by the viscous drag on the aggregates and elastic effects should not play a significant role. Nevertheless, the theory can be extended to quasistatic deformation regime and account for adhesion between MR structures and channel walls, which could be responsible for the elastic behavior of the suspension and for the appearance of the static yield stress.

\section{Conclusions}

In this work, we have proposed a new theoretical model allowing us to explain, for the first time, a strong Bingham rheological behavior of magnetic suspensions in shear flows under a magnetic field parallel to the flow. This model is based on the new concept of magnetically 
induced rotational diffusion caused by long range magnetic interactions between magnetic aggregates present in the suspension. To check our theory, we have carried out experiments on pressure-driven flows of MR fluids in the longitudinal magnetic field at different magnetic field intensities and various particle concentrations of the MR fluids. The main results of this study can be summarized as follows:

1. High level of stress generated in longitudinal magnetic fields is explained by a misalignment of particle aggregates from the streamlines. This misalignment is induced by many-body magnetic interactions between aggregates, which induce stochastic oscillations of the aggregate orientation. Random fluctuations in aggregate orientation are mimicked by an effective rotational diffusion process. The rotary diffusivity, $D_{r}$, is estimated using a random walk model and is found to be proportional to the mean square interaction torque, $\left\langle T_{\text {int }}{ }^{2}\right\rangle \square \mathrm{a}$ net magnetic torque exerted to a given aggregate by all the neighboring aggregates. The theory predicts that the diffusion constant is linear in shear rate, $D_{r}=C \dot{\gamma}$. This comes from the fact that the magnetic aggregates are destructible by shear forces, and, at higher shear rates, they oppose less hydrodynamic resistance to the diffusion process.

2. Our theory predicts that the hydrodynamic stress generated by misaligned aggregates is independent of shear rate in the range of Mason numbers, $\Phi\left\langle e_{1} e_{2}\right\rangle \bigcirc M a=\eta_{0} \dot{\gamma} / f^{m} \bigcirc\left[100\left\langle e_{1} e_{2}\right\rangle\right]^{-1}$, covered in our experiments. Such behavior of the stress is explained by a compromise between two opposite shear rate effects $\square$ enhancement of viscous dissipation, on the one hand, and decrease in the aggregate length with increasing shear rate, on the other hand. This suggests a Bingham behavior of the magnetic suspension in the presence of the longitudinal magnetic field, $\sigma_{12}=\sigma_{Y}+\eta \dot{\gamma}$, with the dynamic yield stress, $\sigma_{Y}$ depending on the magnetic field strength. Such behavior has been well reproduced in our 
experiments, and the experimental values of the yield stress were obtained by a linear extrapolation of the flow curves onto zero shear rate.

3. In experiments, the dynamic yield stress was found to increase almost quadratically with the magnetic field strength $\sigma_{Y} \propto H^{2}$, at magnetic field intensities $H<15 \mathrm{kA} / \mathrm{m}$. The theoretical field dependencies of the yield stress [cf. Eq. (18)] were fitted to the experimental ones by adjusting a single free parameter $\alpha$-interaction coefficient, physically standing for the averaging of the interaction torque over all possible mutual orientations of aggregates. This coefficient was found of the order of unity $(\alpha=1.5)$ and did not depend on the particle volume fraction of the suspension. Both experiments and theory suggest a strong concentration dependence of the yield stress $\left(\sigma_{Y} \propto \Phi^{3}\right.$ at $\Phi<0.3$ and $\left.H<15 \mathrm{kA} / \mathrm{m}\right)$ which is attributed to a strong concentration dependence of the rotary diffusivity. In fact, an increase in the particle volume fraction diminishes the mean lateral spacing between aggregates and, consequently, enhances magnetic interactions between them. This produces stronger fluctuations of the aggregate orientation. At concentrations $0.3<\Phi<0.4$ and $H<15 \mathrm{kA} / \mathrm{m}$, the diffusion stress coming from the random interaction torques became significant and gave a supplementary contribution to the yield stress proportional to $\Phi^{7}$. However, at higher magnetic fields and particle concentrations $(\Phi>0.4$ and $H>15 \mathrm{kA} / \mathrm{m})$, the aggregates are expected to collide with each other. On the one hand, the constraint, that the aggregates cannot cross each other, could attenuate the diffusion process, on the other hand, the aggregates are expected to constantly break during collisions and reform between collisions; this could enhance their rotational diffusion and randomize their orientation.

In perspective, a theoretical or numerical study of collision dynamics of aggregates will be carried out in order to cover a broader range of concentrations and magnetic fields. Applied to 
the shear flows under a magnetic field collinear with the vorticity, our theory will probably be able to explain unexpected stress enhancement observed experimentally in this geometry [Bossis et al. (2002-b)]. In a general sense, the new concept of magnetically induced diffusion may not only be restricted to particular rheological problems, but used for the general purpose of understanding the role of long-range non-hydrodynamic interactions in rotational diffusion observed in concentrated suspensions or granular gases.

\section{Acknowledgements}

We would like to thank Professor Andrey Zubarev for helpful discussions. Biomag (PACA), DynExpert FP7 project $F$ Factories of the Future $\square$ (grant $\mathrm{N}^{\circ}$ 260073) and Cooperation Program between CNRS and BRFFR ( $\mathrm{N}^{\circ} 23178$, France-Belarus) are acknowledged for the financial support. We also appreciated qualified technical assistance of the engineering team (F.Fraisse, L. Constant, J. Hughes, S. Viol) from Malvern Instruments in set-up and maintenance of the Rosand RH-7 capillary rheometer.

\section{References}

Advani S.G., and C.L. Tucker The use of tensors to describe and predict fiber orientation in short fiber composites $\square$ J. Rheol., 31, 751-784 (1987).

Batchelor G.K., $\$ Slender-body theory for particles of arbitrary cross-section in Stokes flow $\square$ J. Fluid. Mech. 44, 419-440 (1970)

Batchelor G.K., $\square$ The stress generated in a non-dilute suspension of elongated particles by pure straining motion $\square$ J. Fluid. Mech. 46, 813-829 (1971)

Bird R.B., O. Hassager, R.C. Armstrong, Ch. F. Curtiss, Dynamics of Polymeric Liquids Volume II. Kinetic Theory, John Wiley and Sons, New York, 1977. 
Bossis G., S. Lacis, A. Meunier, and O. Volkova, Magnetorheological fluids $\square$ J.Magn.Magn.Mater. 252, 224-228 (2002-b).

Bossis G., Volkova O., Lacis S., and Meunier A., in Ferrofluids, $\square$ Magnetorheology: Fluids, Structures and Rheology, edited by S. Odenbach, Springer, Berlin, (2002a).

Carlson J.D., D. M. Catanzarite, and K. A. St. Clair, Commercial magnetorheological fluid devices, $\square$ Int. J. Mod. Phys. B 10, 2857 (1996).

Chaubal Ch. V., and L.G. Leal, $\square$ A closure approximation for liquid-crystalline polymer models based on parametric density estimation $\square$, J.Rheol., 42, 177-201 (1998).

Chung D.H., and T.H. Kwon Improved Model of Orthotropic Closure Approximation for Flow Induced Fiber Orientation $\square$, Polymer Composites, 22, 636-649 (2001).

Cintra J.S., and C.L. Tucker, Orthotropic closure approximations for flow-induced fiber orientation $\square$ J.Rheol. 39, 1095-1122 (1995).

Clercx H. J. H., and G. Bossis, Many-body electrostatic interactions in electrorheological fluids $\square$ Phys. Rev. E 48, 2721\2738 (1993)

Cutillas S., G. Bossis, and A. Cebers, Flow-induced transition from cylindrical to layered patterns in magnetorheological suspensions $\square$, Phys. Rev. E 57, 804 811 (1998)

Doi M. and S.F. Edwards, The theory of polymer dynamics, Oxford University Press, New York, 1986

Férec J., Ausias G., Heuzey M.C. and Carreau P.J., $\square$ Modeling fiber interactions in semiconcentrated fiber suspensions $\square$ J. Rheol. 53, 49-72 (2009).

Folgar, F., and C. L. Tucker, Orientation behavior of fibers in concentrated suspensions, $\square$ J. Reinf. Plast. Compos. 3, 98ロ19 (1984)

Ginder J. M., \Behavior of magnetorheological fluids $\square$, MRS Bull., $2326 \square 29$ (1998).

Ginder J. M., and L. C. Davis, Shear stresses in magnetorheological fluids: Role of magnetic saturation $\square$, Appl. Phys. Lett. 65, 3410-3412 (1994) 
Grosso M., P.L. Maffettone, and F. Dupret $\square$ A closure approximation for nematic liquid crystals based on the canonical distribution subspace theory $\square$ Rheol. Acta, 39, 301-310 (2000).

Hinch E.J., and L.G. Leal, Constitutive equations in suspension mechanics. Part 2. Approximate forms for a suspension of rigid particles affected by Brownian rotations $\square$ J.Fluid Mech. 76, 187-208 (1976)

Jiles D., Introduction to Magnetism and Magnetic Materials, Chapman \& Hill, London, (1991).

Kordonski W. I. and S. D. Jacobs, $\square$ Magnetorheological finishing, $\square$ Int. J. Mod. Phys. B 10, 2837 (1996).

Kuzhir P., G. Bossis, V. Bashtovoi, and O. Volkova, Effect of the orientation of the magnetic field on the flow of magnetorheological fluid. II. Cylindrical channel $\square$ J.Rheol, 47, 1385-1398 (2003).

Kuzhir P., M. T. López-López, and G. Bossis, Abrupt contraction flow of magnetorheological fluids, $\square$ Phys. Fluids 21, 053101 (2009).

Leal L.G. and E.J. Hinch, The rheology of a suspension of nearly spherical particles subject to Brownian rotations $\square$ J. Fluid Mech. 55, 745-765 (1972).

Macosco Ch.W., Rheology. Principles, Measurements, and Applications, Wiley-VCH, Inc., New York; pp. 184-185, 238-256 (1994)

Martin J. E., E. Venturini, G. L. Gulley, and J. Williamson, Using triaxial magnetic fields to create high susceptibility particle composites, $\square$ Phys. Rev. E 69, 021508 (2004).

Martin J.E., R.A. Anderson, Chain model of electrorheology, J.Chem.Phys 104 48144827 (1996).

Otto M., T. Aspelmeier and A. Zippelius, Microscopic dynamics of thin rods, J.Chem.Phys., 124, 144907 (2006) 
Pokrovskiy V.N., Statistical mechanics of diluted suspensions, Nauka, Moscow 1978.

Rosenweig, R. E., Ferrohydrodynamics; Cambridge University Press: New York, 1985.

Shaqfeh, E. S., G. and G. H. Fredrickson, The hydrodynamic stress in a suspension of rods, $\square$ Phys. Fluid A 2, 7-24 (1990).

Shulman Z.P., V.I. Kordonsky, E.A. Zaltsgendler, I.V. Prokhorov, B.M. Khusid and S.A. Demchuk, Structure, physical properties and dynamics of magnetorheological suspensions $\square$, Int J. Multiphase Flow, 12, 935-955 (1986).

Shulman, Z. P., and W. I. Kordonsky, Magnetorheological effect, Nauka i Tehnika, Minsk, 1982 (in Russian)

Stover C. A., D.L. Koch, and C. Cohen, Observations of fibre orientation in simple shear flow of semi-dilute suspensions $\square$ J. Fluid Mech., 238, 277-296 (1992)

Takimoto J., H. Takeda, Y. Masubuchi, and K. Koyama, Stress Rectification in MR Fluids under Tilted Magnetic Field $\square$, Int. J. Mod. Phys. B, 13, 2028-2035 (1999).

Van de Ven G.M., Colloidal hydrodynamics, Academic Press Limited, London (1989).

Volkova O., S. Cutillas, and G. Bossis, Shear Banded Flows and Nematic-toIsotropic Transition in ER and MR Fluids $\square$, Phys. Rev. Lett. 82, $233 \square 236$ (1999)

Zubarev A.Yu., and $\mathrm{L} \mathrm{Yu}$ Iskakova, Rheological properties of ferrofluids with microstructures $\square$, J. Phys.: Condens. Matter 18, S2771 $₫ 2784$ (2006). 